doi:10.13108/2021-13-4-23

\title{
AVERAGING OF RANDOM ORTHOGONAL TRANSFORMATIONS OF DOMAIN OF FUNCTIONS
}

\author{
K.Yu. ZAMANA
}

\begin{abstract}
We consider and study the notions of a random operator, random operatorvalued function and a random semigroup defined on a Hilbert space as well as their averagings. We obtain conditions under which the averaging of a random strongly continuous function is also strongly continuous. In particular, we show that each random strongly continuous contractive operator-valued function possesses a strongly continuous contractive averaging.

We consider two particular random semigroups: a matrix semigroup of random orthogonal transformations of Euclidean space and a semigroup of operators defined on the Hilbert space of functions square integrable on the sphere in the Euclidean space such that these operators describe random orthogonal transformations of the domain these functions. The latter semigroup is called a random rotation semigroup; it can be interpreted as a random walk on the sphere. We prove the existence of the averaging for both random semigroups.

We study an operator-valued function obtained by replacing the time variable $t$ by $\sqrt{t}$ in averaging of the random rotation semigroup. By means of Chernoff theorem, under some conditions, we prove the convergence of the sequence of Feynman-Chernoff iterations of this function to a strongly continuous semigroup describing the diffusion on the sphere in the Euclidean space. In order to do this, we first find and study the derivative of this operatorvalued function at zero being at the same time the generator of the limiting semigroup. We obtain a simple divergence form of this generator. By means of this form we obtain conditions ensuring that this generator is a second order elliptic operator; under these conditions we prove that it is essentially self-adjoint.
\end{abstract}

Keywords: random linear operator, random operator-valued function, averaging, Feynman-Chernoff iterations.

Mathematics Subject Classification: 47B80, 47D06, 60B20

\section{INTRODUCTION}

A problem on constructing and studying averaging of random linear operators and operatorvalued functions is of interest both from the point of view of mathematics and of various applications in problems of statistical physics and quantum mechanics. Here we can mention works [1]-[3], in which analogues of law of large numbers and central limit theorem were established for the products of independent random matrices. In work [4] there were studied limiting characteristics of compositions of random linear transformations. An application of random transformations to describing solutions of evolutionary partial differential equations was provided in work [5]. Averaging of random transformations and application of Chernoff theorem to studying of the limit of the compositions of random semigroups were considered in works [6] 6 .

K.Yu. Zamana, Averaging of random orthogonal transformations of domain of functions.

(C) Zamana K.Yu. 2021.

Submitted March 10, 2021. 
This paper continues studies initiated in work [9], which was devoted to studying the compositions of independent random semigroups $\mathbf{S}(t)$ with values in the group of unitary transformations in the space $L_{2}(D)$ (here $D$ is a spherical layer in the space $\mathbb{R}^{d}$ ) generated by random orthogonal transformations of the space $\mathbb{R}^{d}$ by the formula $\mathbf{S}(t) u(x)=u\left(e^{t \mathbf{A}} x\right)$, where $\mathbf{A}$ is a random skew-symmetric matrix. Assuming that this matrix is uniformly bounded on the probability space and that the averaging of this matrix is zero, in [9] we showed that a sequence of averagings of compositions of independent random operator-valued functions of form $\mathbf{S}(\sqrt{t})$ converged to a semigroup resolving some initial boundary value problem for an evolutionary second order partial differential equation being an analogue of diffusion equation.

In [9], an inaccuracy was made while studying the differential operator being a generator of this limiting semigroup. The matter is that this operator is not uniformly elliptic if we consider it on the space of the functions defined on the spherical layer. This is due to the fact that the spheres are invariant manifolds for random orthogonal transformations and this is why thee are also invariant for the averaging. As a result, a degeneracy of the operator occurs along the radial direction and this breaks the ellipticity condition. However, as it we show in Theorem 4.4 of the present paper, if we consider this operator on a space of functions defined on a sphere, then the ellipticity can be recovered. Thus, this inaccuracy can be easily corrected and it makes no influence on the results and conclusions made in [9].

The aim of the present work is to make a passage to the limit for a sequence of FeynmanChernoff iterations of operator-valued functions arisen in averaging an operator-valued function $\mathbf{S}(\sqrt{t})$ under the assumption that the random matrix $\mathbf{A}$ is not necessarily bounded but possesses a finite second moment. This passage to the limit is made by using the Chernoff theorem. Because of this, in the present paper we justify the applicability of Chernoff theorem to the considered sequence of iterations.

The scheme of studies presented in this paper is as follows. First we recall some needed notions, namely, the notion of random operator and random operator-valued function, the notion on their averaging and list their main properties, see Statements 2.1, 2.2, 2.3 and Corollaries 2.1, 2.2. We obtain sufficient conditions ensuring a strong continuity of the averaging of a random operator-valued function, the values of which are operators defined on a separable Hilbert space, see Statement 2.4. Then we provide some auxiliary estimates and results concerning a random matrix exponent $e^{t \mathbf{A}}$, see Statement 3.1. Corollary 3.1 and Theorem 3.1. In Theorem 4.1 and Corollary 4.1 we justify the existence of a strong continuous averaging of a random operator-valued function $\mathbf{S}(\sqrt{t})$ acting on the spaces $L_{2}\left(\mathbb{R}^{d}\right)$ and $L_{2}\left(\mathbb{S}^{d-1}\right)$ and performing random orthogonal transformations in the space $\mathbb{R}^{d}$. Finally, in Theorems 4.2 and 4.3 . we obtain a derivative of this averaging at zero under the assumption that the second moment of the random matrix $\mathbf{A}$ is finite, while in Theorem 4.4 and its Corollary 4.2 we establish the convergence of the Feynman-Chernoff iterations of this averaging in the space $L_{2}\left(\mathbb{S}^{d-1}\right)$ to the semigroup generated by the derivative.

Thus, in the paper we propose a method of constructing averagings for random orthogonal transformations of the domain of a function leading us to constructing semigroups describing the diffusion on a sphere.

\section{PRELIMINARIES AND TERMINOLOGY}

Let $\mathcal{H}$ be a Hilbert space (finite- or infinite-dimensional) over the field $\mathbb{R}$ or $\mathbb{C}$ with a scalar product $\langle\cdot, \cdot\rangle$ and the induced norm $\|\cdot\|_{\mathcal{H}}, B(\mathcal{H})$ be a normed space of linear bounded operators $A: \mathcal{H} \rightarrow \mathcal{H}$ with the operator norm $\|\cdot\|_{B(\mathcal{H})}$ and the identity mapping $I,(\Omega, \mathcal{F}, \mathbb{P})$ be a probability space. 
Definition 2.1. A mapping $\mathbf{A}: \Omega \rightarrow B(\mathcal{H})$ with values $\mathbf{A}_{\omega} \in B(\mathcal{H})$, where $\omega \in \Omega$, is called a random operator on $\mathcal{H}$ if the functions $\xi(\cdot)=\left\langle\mathbf{A}_{(\cdot)} u, v\right\rangle$ are $(\Omega, \mathcal{F})$-measurable, that is, are random variables, for all $u \in \mathcal{H}$ and $v \in \mathcal{H}$.

If $\mathbf{A}: \Omega \rightarrow B(\mathcal{H})$ is a random operator, then each vector $u \in \mathcal{H}$ induces a mapping $\mathbf{A}_{(\cdot)} u: \Omega \rightarrow \mathcal{H}$, which we shortly denote $\mathbf{A} u$. In the same way, for all vectors $u \in \mathcal{H}$ and $v \in \mathcal{H}$ the random variable $\left\langle\mathbf{A}_{(\cdot)} u, v\right\rangle$ is denoted as $\langle\mathbf{A} u, v\rangle$. A sequence of random operators indexed by natural numbers is denoted by $(\mathbf{A})_{n}$ in order to distinguish it from the writing $\mathbf{A}_{\omega}$.

A random operator in Definition 2.1 is a mapping measurable with respect to a standard base of the weak operator topology, that is, the pre-image of each element in the standard base of the weak operator topology in the space $B(\mathcal{H})$ under the mapping $\mathbf{A}$ belongs to $\mathcal{F}$. For each $u \in \mathcal{H}$, the mapping $\mathbf{A} u$ induced by this random operator is measurable with respect to the standard base of the weak topology of the space $\mathcal{H}$.

We mention some properties of random operator, which will be used in our work:

1. If $\mathbf{A}$ and $\mathbf{B}$ are random operators, then for all scalars $\alpha$ and $\beta$ the mapping $\alpha \mathbf{A}+\beta \mathbf{B}$ is also a random operator.

2. Let a sequence of random operators $\left\{(\mathbf{A})_{n}\right\}_{n=1}^{\infty}$ converge to the mapping $\mathbf{A}: \Omega \rightarrow B(\mathcal{H})$ in the weak operator topology of the space $B(\mathcal{H})$ almost everywhere on $\Omega$. Then $\mathbf{A}$ is also a random operator.

3. Let $\mathcal{H}$ be a Hilbert space, $\mathcal{D}$ be an everywhere dense subspace in $\mathcal{H}$. Let a mapping A : $\Omega \rightarrow B(\mathcal{H})$ be such that for all $u$ and $v$ in $\mathcal{D}$ the function $\langle\mathbf{A} u, v\rangle$ is a random variable. Then $\mathbf{A}$ is a random operator on $\mathcal{H}$.

The latter property allows us to check the measurability of the mapping $\mathbf{A}$ only on a dense subspace.

Definition 2.2. An averaging (or expectation, or integral over $\Omega$ ) of a random operator $\mathbf{A}: \Omega \rightarrow B(\mathcal{H})$ is an operator $\mathrm{MA} \in B(\mathcal{H})$ such that

$$
\langle(\mathrm{MA}) u, v\rangle=\mathrm{M}\langle\mathbf{A} u, v\rangle \quad \forall u \in \mathcal{H} \quad \forall v \in \mathcal{H},
$$

where $\mathrm{M}$ in the right hand side of identity (2.1) denotes the expectation, that is, the integral over the probability space $(\Omega, \mathcal{F}, \mathbb{P})$.

The averaging possesses the following properties:

1. If it exists, then the averaging of a random operator is unique.

2. If random operators $\mathbf{A}$ and $\mathbf{B}$ possess averagings, then for all scalars $\alpha$ and $\beta$ the random operator $\alpha \mathbf{A}+\beta \mathbf{B}$ also possesses the averaging and

$$
\mathrm{M}(\alpha \mathbf{A}+\beta \mathbf{B})=\alpha \mathrm{MA}+\beta \mathrm{MB} .
$$

We shall also need the following sufficient condition for the existence of the averaging of a random operator.

Statement 2.1 (9]). Let A be a random operator on the Hilbert space $\mathcal{H}$ such that $\|\mathbf{A}\|_{B(\mathcal{H})} \leqslant \xi$, where $\xi: \Omega \rightarrow[0 ;+\infty)$ is a random variable with a finite expectation. Then A possesses an averaging $\mathrm{MA} \in B(\mathcal{H})$ and

$$
\|\mathrm{MA}\|_{B(\mathcal{H})} \leqslant \mathrm{M} \xi .
$$

In particular, if the function $\|\mathbf{A}\|_{B(\mathcal{H})}$ is a random variable with a finite expectation, then $\mathbf{A}$ possesses an averaging and

$$
\|\mathrm{MA}\|_{B(\mathcal{H})} \leqslant \mathrm{M}\|\mathbf{A}\|_{B(\mathcal{H})} .
$$

We recall that an operator $A \in B(\mathcal{H})$ is called contractive if $\|A\|_{B(\mathcal{H})} \leqslant 1$; a random operator is called a random contractive operator if all its values are contractive operator. Applying Statement 2.1 to such random operator, we immediately obtain the following statement. 
Corollary 2.1. Each random contractive operator on a Hilbert space possesses an averaging, which is also a contractive operator.

In the case when the space $\mathcal{H}$ is separable, the measurability of a random operator in the weak operator topology implies the measurability in the strong and uniform operator topology.

Statement 2.2. Let $\mathcal{H}$ be a separable Hilbert space, $\mathbf{A}: \Omega \rightarrow B(\mathcal{H})$ be a random operator. Then

a) $\|\mathbf{A} u\|_{\mathcal{H}}$ is a random variable for each $u \in \mathcal{H}$;

b) $\|\mathbf{A}\|_{B(\mathcal{H})}$ is a random variable.

If $\mathbf{A}$ possesses the averaging MA, then $\|(\mathrm{MA}) u\|_{\mathcal{H}} \leqslant \mathrm{M}\|\mathbf{A} u\|_{\mathcal{H}}$ for each $u \in \mathcal{H}$ and $\|\mathbf{M A}\|_{B(\mathcal{H})} \leqslant \mathrm{M}\|\mathbf{A}\|_{B(\mathcal{H})} ;$ at that $\mathrm{M}\|\mathbf{A} u\|_{\mathcal{H}}$ and $\mathrm{M}\|\mathbf{A}\|_{B(\mathcal{H})}$ can be infinite.

Proof. Let $\left\{v_{n}\right\}_{n=1}^{\infty} \subset \mathcal{H}$ be a countable everywhere dense in $\mathcal{H}$ set of vectors. Without loss of generality we can assume that these vectors are non-zero. Then the vectors $e_{n}=\frac{v_{n}}{\left\|v_{n}\right\|_{\mathcal{H}}}$ form a countable subset of a unit sphere in the space $\mathcal{H}$ dense on this sphere. Therefore,

$$
\|\mathbf{A} u\|_{\mathcal{H}}=\sup _{n \in \mathbb{N}}\left|\left\langle\mathbf{A} u, e_{n}\right\rangle\right|
$$

for each $u \in \mathcal{H}$

Since $\mathbf{A}$ is a random operator, then all functions $\left|\left\langle\mathbf{A} u, e_{n}\right\rangle\right|$ are measurable and this is why $\|\mathbf{A} u\|_{\mathcal{H}}$ is measurable as a point-wise supremum of a countable set of measurable functions.

In the same way, in view of the just proven fact, the measurability of $\|\mathbf{A}\|_{B(\mathcal{H})}$ is implied by the identity

$$
\|\mathbf{A}\|_{B(\mathcal{H})}=\sup _{n \in \mathbb{N}}\left\|\mathbf{A} e_{n}\right\|_{\mathcal{H}}
$$

Suppose now that $\mathbf{A}$ has an averaging MA. In view of the just proven fact we get

$$
\begin{aligned}
\|(\mathrm{MA}) u\|_{\mathcal{H}} & =\sup _{\|v\|_{\mathcal{H}}=1}|\langle(\mathrm{MA}) u, v\rangle|=\sup _{\|v\|_{\mathcal{H}}=1}|\mathrm{M}\langle\mathbf{A} u, v\rangle| \\
& \leqslant \sup _{\|v\|_{\mathcal{H}}=1} \mathrm{M}|\langle\mathbf{A} u, v\rangle| \leqslant \sup _{\|v\|_{\mathcal{H}}=1} \mathrm{M}\|\mathbf{A} u\|_{\mathcal{H}}\|v\|_{\mathcal{H}}=\mathrm{M}\|\mathbf{A} u\|_{\mathcal{H}},
\end{aligned}
$$

and similarly, again by the just proven fact,

$$
\|\mathrm{MA}\|_{B(\mathcal{H})}=\sup _{\|u\|_{\mathcal{H}}=1}\|(\mathrm{MA}) u\|_{\mathcal{H}} \leqslant \sup _{\|u\|_{\mathcal{H}}=1} \mathrm{M}\|\mathbf{A} u\|_{\mathcal{H}} \leqslant \sup _{\|u\|_{\mathcal{H}}=1} \mathrm{M}\|\mathbf{A}\|_{B(\mathcal{H})}\|u\|_{\mathcal{H}}=\mathrm{M}\|\mathbf{A}\|_{B(\mathcal{H})} .
$$

This completes the proof.

Remark 2.1. Statement 2.2 remains true also in the case when the Hilbert space $\mathcal{H}$ is not separable but if at the same time the probability space $(\Omega, \mathcal{F}, \mathbb{P})$ is discrete. Indeed, the separability condition in Statement 2.2 is needed only to establish the measurability of $\|\mathbf{A} u\|_{\mathcal{H}}$ and $\|\mathbf{A}\|_{B(\mathcal{H})}$ and this holds immediately for a discrete probability space.

For the averaging operation we can formulate an analogue of Lebesgue's dominated convergence theorem.

Statement 2.3. Let a sequence of random operators $\left\{(\mathbf{A})_{n}\right\}_{n=1}^{\infty}$ converge to a random operator $\mathbf{A}$ in the weak operator topology of the space $B(\mathcal{H})$ almost everywhere on $\Omega$, and $\left\|(\mathbf{A})_{n}\right\|_{B(\mathcal{H})} \leqslant \xi$ for all $n \in \mathbb{N}$, where $\xi: \Omega \rightarrow[0 ;+\infty)$ is a random variable with a finite expectation. Then the random operators $(\mathbf{A})_{n}$ and $\mathbf{A}$ have averagings and $\mathrm{M}(\mathbf{A})_{n}$ converge to MA in the weak operator topology of the space $B(\mathcal{H})$.

Proof. By inequality $\left\|(\mathbf{A})_{n}\right\|_{B(\mathcal{H})} \leqslant \xi$, Statement 2.1 implies that the random operators $(\mathbf{A})_{n}$ possess averagings. The same inequality implies that

$$
\left|\left\langle(\mathbf{A})_{n} u, v\right\rangle\right| \leqslant \xi\|u\|_{\mathcal{H}}\|v\|_{\mathcal{H}} \quad \forall u \in \mathcal{H} \quad \forall v \in \mathcal{H} .
$$


Passing in (2.2) to the limit as $n \rightarrow \infty$ and employing the convergence of $\left\{(\mathbf{A})_{n}\right\}_{n=1}^{\infty}$ to $\mathbf{A}$ in the weak operator topology, we obtain that $|\langle\mathbf{A} u, v\rangle| \leqslant \xi\|u\|_{\mathcal{H}}\left\|_{v}\right\|_{\mathcal{H}}$ almost everywhere. Taking the supremum in this inequality with respect to $\|v\|_{\mathcal{H}}=1$ and $\|u\|_{\mathcal{H}}=1$, we obtain $\|\mathbf{A}\|_{B(\mathcal{H})} \leqslant \xi$ almost everywhere and by Statement 2.1 this implies the existence of the averaging of $\mathbf{A}$. For fixed $u \in \mathcal{H}$ and $v \in \mathcal{H}$ a sequence of random variables $\left\langle(\mathbf{A})_{n} u, v\right\rangle$ converges to $\langle\mathbf{A} u, v\rangle$ almost everywhere by $(2.2)$, satisfies all assumptions of the Lebesgue's dominated convergence theorem and this is why

$$
\lim _{n \rightarrow \infty}\left\langle\mathrm{M}(\mathbf{A})_{n} u, v\right\rangle=\lim _{n \rightarrow \infty} \mathrm{M}\left\langle(\mathbf{A})_{n} u, v\right\rangle=\mathrm{M}\langle\mathbf{A} u, v\rangle=\langle(\mathrm{MA}) u, v\rangle .
$$

This completes the proof.

Remark 2.2. For a case of a separable $\mathcal{H}$ or a discrete $(\Omega, \mathcal{F}, \mathbb{P})$, analogues of Statement 2.3 hold true, in which the weak topology is replaced by the strong or uniform one.

The set of all mappings $F: \mathbb{R}_{+} \rightarrow B(\mathcal{H})$, where $\mathbb{R}_{+}=[0 ;+\infty)$, is traditionally denoted by $B(\mathcal{H})^{\mathbb{R}_{+}}$and each its element is called an operator-valued function; in what follows we shall often omit the adjective "operator-valed" in the cases, when this does not lead to misunderstanding. Let $C_{s}\left(\mathbb{R}_{+}, B(\mathcal{H})\right)$ be a topological vector space consisting of operator-valued functions $F: \mathbb{R}_{+} \rightarrow B(\mathcal{H})$ continuous with respect to a strong operator topology of the space $B(\mathcal{H})$ (such functions are called strongly continuous); a topology $\tau_{s}$ in $C_{s}\left(\mathbb{R}_{+}, B(\mathcal{H})\right)$ is induced by a family of semi-norms $\Phi_{T, u}(F)=\sup _{t \in[0 ; T]}\|F(t) u\|_{\mathcal{H}}$ for all $T>0$ and $u \in \mathcal{H}$. We note that if $F$ is an operator-valued function and $F_{n} \in C_{s}\left(\mathbb{R}_{+}, B(\mathcal{H})\right)$ for each $n \in \mathbb{N}$, then

$$
\begin{aligned}
& F \in C_{s}\left(\mathbb{R}_{+}, B(\mathcal{H})\right) \Leftrightarrow \quad \lim _{t \rightarrow t_{0}}\left\|F(t) u-F\left(t_{0}\right) u\right\|_{\mathcal{H}}=0 \quad \forall t_{0} \geqslant 0 \quad \forall u \in \mathcal{H}, \\
& F_{n} \stackrel{\tau_{s}}{\longrightarrow} F \quad \Leftrightarrow \quad \lim _{n \rightarrow \infty} \sup _{t \in[0 ; T]}\left\|F_{n}(t) u-F(t) u\right\|_{\mathcal{H}}=0 \quad \forall T>0 \quad \forall u \in \mathcal{H} .
\end{aligned}
$$

An operator-valued function $S: \mathbb{R}_{+} \rightarrow B(\mathcal{H})$ is called an operator semigroup (or simply semigroup) if

$$
S(0)=I, \quad S\left(t_{1}+t_{2}\right)=S\left(t_{1}\right) S\left(t_{2}\right) \quad \forall t_{1}, t_{2} \in[0 ;+\infty) .
$$

Definition 2.3. A mapping $\mathbf{F}: \Omega \rightarrow B(\mathcal{H})^{\mathbb{R}_{+}}$with values $\mathbf{F}_{\omega}: \mathbb{R}_{+} \rightarrow B(\mathcal{H})$, where $\omega \in \Omega$, is called a random operator-valued function (or simply random function) if $\mathbf{F}_{(\cdot)}(t)$ is a random operator for each $t \geqslant 0$. If at the same time $\mathbf{F}_{\omega}$ is an operator semigroup for each $\omega \in \Omega$, then the mapping $\mathbf{F}$ is also called a random operator semigroup or simply a random semigroup).

Let us make some important remarks about the notations related with the random function F:

- the value of the random function $\mathbf{F}$ on an event $\omega \in \Omega$ is denoted by $\mathbf{F}_{\omega}$ and this is an operator-valued function;

- for each $t \geqslant 0$ the mapping $\mathbf{F}_{(\cdot)}(t): \Omega \rightarrow B(\mathcal{H})$, being a random operator by Definition 2.3 , is denoted by $\mathbf{F}(t)$; such notation should not lead to a misunderstanding since according to the previous item, the value of the random function $\mathbf{F}$ on an event $\omega \in \Omega$ is denoted by $\mathbf{F}_{\omega}$ instead of $\mathbf{F}(\omega)$;

- the value of a random operator $\mathbf{F}(t)$ on an event $\omega \in \Omega$, being an operator, is denoted by $\mathbf{F}_{\omega}(t)$;

- the remark after Definition 2.1 concerns also the random operator $\mathbf{F}(t)$, that is, for each $u \in \mathcal{H}$ the mapping $\mathbf{F}_{(\cdot)}(t) u$ is denoted by $\mathbf{F}(t) u$.

If all values of a random operator-valued function are strongly continuous functions, then such random function is called a random strongly continuous operator-valued function. 
Definition 2.4. An averaging of a random operator-valued function $\mathbf{F}$ is a function $\mathrm{MF}: \mathbb{R}_{+} \rightarrow B(\mathcal{H})$ such that $(\mathrm{MF})(t)$ is an averaging of a random operator $\mathbf{F}(t)$ for each $t \geqslant 0$, that is, $(\mathrm{MF})(t)=\mathrm{M}(\mathbf{F}(t))$.

In order to prove the existence of averagings of random operator-valued functions, we use Statement 2.1 and its Corollary 2.1. However, an averaging of a random strongly continuous function is not necessary a strongly continuous function. This is why it is useful to consider the following modification of Statement 2.1 allowing us to guarantee a strong continuity of the averaging.

Statement 2.4. Let $\mathcal{H}$ be a separable Hilbert space, $\mathbf{F}: \Omega \rightarrow C_{s}\left(\mathbb{R}_{+}, B(\mathcal{H})\right)$ be a random strongly continuous operator-valued function, which satisfies the following condition: for each $T>0$ there exists a random variable $\xi_{T}$ with a finite expectation such that $\|\mathbf{F}(t)\|_{B(\mathcal{H})} \leqslant \xi_{T}$ on $[0 ; T]$. Then $\mathbf{F}$ has a strongly continuous averaging $\mathrm{MF} \in C_{s}\left(\mathbb{R}_{+}, B(\mathcal{H})\right)$ and $\|(\mathrm{MF})(t)\|_{B(\mathcal{H})} \leqslant$ $\mathrm{M} \xi_{T}$ on each segment $[0 ; T]$.

Proof. The existence of averaging $\mathrm{MF} \in B(\mathcal{H})^{\mathbb{R}_{+}}$and the estimate for it are implied immediately by Statement 2.1. We just need to prove the strong continuity.

According to Statement 2.2, for each $u \in \mathcal{H}$ we have

$$
\left\|(\mathrm{MF})(t) u-(\mathrm{MF})\left(t_{0}\right) u\right\|_{\mathcal{H}}=\left\|\mathrm{M}\left(\mathbf{F}(t)-\mathbf{F}\left(t_{0}\right)\right) u\right\|_{\mathcal{H}} \leqslant \mathrm{M}\left\|\mathbf{F}(t) u-\mathbf{F}\left(t_{0}\right) u\right\|_{\mathcal{H}} .
$$

Since $\mathbf{F}$ is a random strongly continuous function, then $\left\|\mathbf{F}(t) u-\mathbf{F}\left(t_{0}\right) u\right\|_{\mathcal{H}}$ converges to zero as $t \rightarrow t_{0}$ point-wise on $\Omega$. At that, if $t \in\left[t_{0}-1 ; t_{0}+1\right] \cap \mathbb{R}_{+}$, then

$$
\left\|\mathbf{F}(t) u-\mathbf{F}\left(t_{0}\right) u\right\|_{\mathcal{H}} \leqslant\left(\|\mathbf{F}(t)\|_{B(\mathcal{H})}+\left\|\mathbf{F}\left(t_{0}\right)\right\|_{B(\mathcal{H})}\right)\|u\|_{\mathcal{H}} \leqslant 2\|u\|_{\mathcal{H}} \cdot \xi_{t_{0}+1},
$$

that is, $\left\|\mathbf{F}(t) u-\mathbf{F}\left(t_{0}\right) u\right\|_{\mathcal{H}}$ is majorized by an integrable random variable $2\|u\|_{\mathcal{H}} \cdot \xi_{t_{0}+1}$ in the vicinity of $t_{0}$. Therefore, by the Lebesgue's dominated convergence theorem, the right hand side of the inequality in (2.3) tends to zero as $t \rightarrow t_{0}$ and this is why the left-hand side in (2.3) tends to zero as well. The proof is complete.

Remark 2.3. As with Statement 2.2, the condition of the separability of $\mathcal{H}$ in Statement 2.4 can be replaced by the condition of discreteness of the probability space $(\Omega, \mathcal{F}, \mathbb{P})$.

If all values of the operator-valued functions are contractive operators, we call such function contractive; if all values of a random operator-valued function are contractive functions, we call such random function a random contractive function. By Statement 2.4 we immediately obtain the following corollary.

Corollary 2.2. Each random strongly continuous contractive operator-valued function acting on a separable Hilbert space possesses an averaging, which itself is a strongly continuous contractive operator-valued function.

Definition 2.5. A function $F \in C_{s}\left(\mathbb{R}_{+}, B(\mathcal{H})\right)$ is Chernoff equivalent to an operator semigroup $U \in C_{s}\left(\mathbb{R}_{+}, B(\mathcal{H})\right)$ if the sequence of Feynman-Chernoff iterations $(F(t / n))^{n}$ of the function $F$ converges to the semigroup $U$ in the topology of the space $C_{s}\left(\mathbb{R}_{+}, B(\mathcal{H})\right)$.

The following theorem provides sufficient conditions of the Chernoff equivalence to an operator semigroup, see [10] and [11].

Theorem (Chernoff). Let a function $F \in C_{s}\left(\mathbb{R}_{+}, B(\mathcal{H})\right)$ be such that $F(0)=I$ and $\|F(t)\|_{B(\mathcal{H})} \leqslant e^{\alpha t}$ for some $\alpha \geqslant 0$. If the closure of the operator $F^{\prime}(0)$ is a generator of the semigroup $U \in C_{s}\left(\mathbb{R}_{+}, B(\mathcal{H})\right)$, then the function $F$ is Chernoff equivalent of the semigroup $U$. 
The checking of the assumptions of this theorem for the averaging of a random operatorvalued function $\mathbf{T}(t) u=u\left(e^{\sqrt{t} \mathbf{A}} x\right)$ takes an essential part of the calculations in the present paper.

\section{PROPERTIES OF AVERAGINGS OF RANDOM MATRICES AND MATRIX SEMIGROUPS}

In this section we obtain certain auxiliary results related with random matrices and their averagings.

If we take $\mathbb{R}^{d}$ as $\mathcal{H}$, where $d \in \mathbb{N}$, then $B\left(\mathbb{R}^{d}\right)$ can be considered as a normed space $\mathcal{M}_{d}(\mathbb{R})$ of square matrices of order $d$ with the spectral norm. Moreover, there exists an isomorphism between the spaces $B\left(\mathbb{R}^{d}\right)$ and $\mathcal{M}_{d}(\mathbb{R})$, which maps each linear operator into its matrix in the standard basis of the space $\mathbb{R}^{d}$. In view of this, a random operator $\mathbf{A}: \Omega \rightarrow B\left(\mathbb{R}^{d}\right)$ is called a random matrix, and its values are identified with the corresponding matrices. An entry $\mathbf{a}_{i j}$ of a random matrix $\mathbf{A}$ is a random variable $\left(\mathbf{A} e_{j}, e_{i}\right)$, where $\left\{e_{i}\right\}_{i=1}^{d}$ is the standard basis in the space $\mathbb{R}^{d}$. In the case $\mathcal{H}=\mathbb{C}^{d}$ we proceed in the same way.

Hereinafter the scalar product in the space $\mathbb{R}^{d}$ is denoted by the round brackets $(\cdot, \cdot)$, the Euclidean norm of a vector $x \in \mathbb{R}^{d}$ is denoted by the symbol $|x|$, while the spectral norm of the matrix $A \in B\left(\mathbb{R}^{d}\right)$, being also its operator norm, is denoted by $\|A\|$ without indicating the subscript $B\left(\mathbb{R}^{d}\right)$.

A finite-dimensional property of the spaces $\mathbb{R}^{d}$ and $B\left(\mathbb{R}^{d}\right)$ allows us to make stronger conclusions on the measurability and averagings of random matrices in comparison with Statement 2.2,

1. The mapping $\mathbf{A}: \Omega \rightarrow B\left(\mathbb{R}^{d}\right)$ is a random matrix if and only if its entries are random variables.

2. If $\mathbf{A}$ is a random matrix, then for each $x \in \mathbb{R}^{d}$ the mapping $\mathbf{A} x: \Omega \rightarrow \mathbb{R}^{d}$ is a random vector.

3. The random matrix $\mathbf{A}$ possesses an averaging if and only if the random variable $\|\mathbf{A}\|$ has a finite expectation. At that, the entries of the matrix MA are expectations of corresponding entries of $\mathbf{A}$.

By the product of random matrices $\mathbf{A}$ and $\mathbf{B}$ we mean the mapping $\mathbf{A B}: \Omega \rightarrow B\left(\mathbb{R}^{d}\right)$, which maps each $\omega \in \Omega$ into the matrix $\mathbf{A}_{\omega} \mathbf{B}_{\omega}$. The product of random matrices is also a random matrix.

Let $\mathbf{A}$ be a random matrix. We consider a matrix exponent:

$$
e^{t \mathbf{A}}=\sum_{k=0}^{\infty} \frac{t^{k} \mathbf{A}^{k}}{k !} \quad \forall t \in \mathbb{R}_{+} .
$$

We observe that $e^{t \mathbf{A}}$ is a random continuous matrix semigroup. And vice versa, if $\mathbf{S}: \Omega \rightarrow$ $C_{s}\left(\mathbb{R}_{+}, B\left(\mathbb{R}^{d}\right)\right)$ is a random continuous matrix semigroup, then there exists a random matrix A such that $\mathbf{S}(t)=e^{t \mathbf{A}}$. We call this random matrix a random generator (or simply generator) of the random semigroup $\mathbf{S}$.

For the purposes in the next section we are interested in a random continuous matrix semigroup performing random orthogonal transformations (rotations) in the space $\mathbb{R}^{d}$. Such random semigroup is called orthogonal. In view of the facts said above, it is easy to observe that the generator of each random continuous orthogonal matrix semigroup is a random skew-symmetric matrix. And vice versa, each random skew-symmetric matrix generates a random continuous orthogonal matrix semigroup.

Applying Corollary 2.2 to a random continuous orthogonal matrix function, we arrive at the following statement.

Corollary 3.1. Each random continuous orthogonal matrix function possesses an averaging, which is a continuous contractive matrix function. 
We shall also need some properties of the spectral norm of a skew-symmetric matrix $A$ :

1) $\left\|A^{n}\right\|=\|A\|^{n}$;

2) $\left\|A^{k}\right\| \leqslant\left\|A^{n}\right\|+1$ for all $k$ from 1 to $n$.

This implies the following properties of a random skew-symmetrix matrix A:

1. A random matrix $\mathbf{A}^{n}$ possesses an averaging if and only if the random variable $\|\mathbf{A}\|^{n}$ possesses a finite expectation.

2. If $\mathbf{A}^{n}$ possesses an averaging, then random matrices $\mathbf{A}^{k}$ for all $k$ from 1 to $n$ possess averagings.

Finally we obtain some useful estimates for the remainder of series 3.1$)$ in the case when the matrix $A$ is skew-symmetric.

Statement 3.1. Let $A$ be a skew-symmetric matrix, $n \in \mathbb{N} \cup\{0\}, t \geqslant 0$. Then the following inequalities hold:

$$
\text { a) }\left\|e^{t A}-\sum_{k=0}^{n} \frac{t^{k} A^{k}}{k !}\right\| \leqslant \frac{t^{n+1}\left\|A^{n+1}\right\|}{(n+1) !} ; \quad \text { b) }\left\|e^{t A}-\sum_{k=0}^{n} \frac{t^{k} A^{k}}{k !}\right\| \leqslant \frac{2 t^{n}\left\|A^{n}\right\|}{n !} .
$$

Proof. Inequality a) is implied by the Taylor formula of order $n$ at the point $t=0$ with the remainder in the Lagrange form [12] applied to the matrix function $e^{t A}$. Indeed, $\left(e^{t A}\right)^{(k)}(0)=A^{k}$ for all $k$ from 0 to $n$ and since the matrix $e^{t A}$ is orthogonal, then

$$
\left\|\left(e^{t A}\right)^{(n+1)}\right\|=\left\|A^{n+1} e^{t A}\right\| \leqslant\left\|A^{n+1}\right\| \cdot\left\|e^{t A}\right\|=\left\|A^{n+1}\right\| .
$$

Inequality b) is implied by inequality a):

$$
\left\|e^{t A}-\sum_{k=0}^{n} \frac{t^{k} A^{k}}{k !}\right\| \leqslant\left\|e^{t A}-\sum_{k=0}^{n-1} \frac{t^{k} A^{k}}{k !}\right\|+\left\|\frac{t^{n} A^{n}}{n !}\right\| \leqslant \frac{2 t^{n}\left\|A^{n}\right\|}{n !} .
$$

Theorem 3.1. Let $\mathbf{A}$ be a random skew-symmetric matrix such that $\mathbf{A}^{n}$ possesses an averaging for some $n \in \mathbb{N}$. Then

$$
\lim _{t \rightarrow 0+} \frac{1}{t^{n}}\left\|\mathrm{M}\left(e^{t \mathbf{A}}\right)-\sum_{k=0}^{n} \frac{t^{k} \mathrm{M}\left(\mathbf{A}^{k}\right)}{k !}\right\|=0 .
$$

In particular, if $\mathrm{M}\left(\mathbf{A}^{k}\right)=0$ for all $k$ from 1 to $n-1$, then

$$
\left(\mathrm{M}\left(e^{\sqrt[n]{t} \mathbf{A}}\right)\right)^{\prime}(0)=\frac{\mathrm{M}\left(\mathbf{A}^{n}\right)}{n !}
$$

and the matrix function $\mathrm{M}\left(e^{\sqrt[n]{t} \mathbf{A}}\right)$ is Chernoff equivalent to the matrix semigroup $e^{\frac{t \mathrm{M}\left(\mathbf{A}^{n}\right)}{n !}}$.

Proof. For $t>0$ we denote:

$$
\mathbf{B}(t)=\frac{1}{t^{n}}\left(e^{t \mathbf{A}}-\sum_{k=0}^{n} \frac{t^{k} \mathbf{A}^{k}}{k !}\right) .
$$

The random matrices $\mathbf{A}^{k}$ for $k$ from 1 to $n$ as well as a random continuous orthogonal matrix semigroup $e^{t \mathbf{A}}$ possess averagings and this is why $\mathbf{B}(t)$ is a random matrix for each $t>0$ possessing an averaging:

$$
(\mathrm{MB})(t)=\frac{1}{t^{n}}\left(\mathrm{M}\left(e^{t \mathbf{A}}\right)-\sum_{k=0}^{n} \frac{t^{k} \mathrm{M}\left(\mathbf{A}^{k}\right)}{k !}\right)
$$


Applying inequality a) from Statement 3.1, we obtain:

$$
\|\mathbf{B}(t)\| \leqslant \frac{t\left\|\mathbf{A}^{n+1}\right\|}{(n+1) !} \stackrel{t \rightarrow 0+}{\longrightarrow} 0,
$$

that is, a random matrix $\mathbf{B}(t)$ converges to the zero matrix as $t \rightarrow 0+$ pointwise on $\Omega$. On the other hand, according to inequality b) of Statement 3.1

$$
\|\mathbf{B}(t)\| \leqslant \frac{2\left\|\mathbf{A}^{n}\right\|}{n !}
$$

and by our assumptions the random variable $\left\|\mathbf{A}^{n}\right\|$ possesses a finite expectation, that is, the norm of $\mathbf{B}(t)$ is majorized by an integrable random variable in a right neighbourhood of the zero. Then by Statement 2.3. (MB) $(t)$ converges to the zero matrix as $t \rightarrow 0+$ and this completes the proof.

\section{AVERAGING OF RANDOM ROTATION OPERATORS}

Let $\mathcal{H}=L_{2}\left(\mathbb{R}^{d}\right)$ be a Hilbert space of functions $u: \mathbb{R}^{d} \rightarrow \mathbb{C}$ square integrable with respect to the standard Lebesgue measure $\mu$ on $\mathbb{R}^{d}$. Let $\mathbf{A}: \Omega \rightarrow B\left(\mathbb{R}^{d}\right)$ be a random skew-symmetric matrix of order $d$.

In this section we consider random operators $\mathbf{S}(t) u(x)=u\left(e^{t \mathbf{A}} x\right)$ and $\mathbf{T}(t)=\mathbf{S}(\sqrt{t})$ performing random orthogonal transformations (rotations) of the domain of a function $u \in \mathcal{H}$ and we study their averagings. A central result of this section is the proof of the Chernoff equivalence of the averaging MT to some operator semigroup describing a diffusion on a $(d-1)$-dimensional sphere $\mathbb{S}^{d-1}$ under the assumptions $\mathrm{MA}=0$ and $\mathrm{M}\left(\mathbf{A}^{2}\right) \in B\left(\mathbb{R}^{d}\right)$.

4.1. Well-definiteness and existence of averaging. For each $\omega \in \Omega$ and $t \geqslant 0$ we consider an operator $\mathbf{S}_{\omega}(t) \in B(\mathcal{H})$, which maps each function $u \in \mathcal{H}$ into the function $u\left(e^{t \mathbf{A}_{\omega}}(\cdot)\right)$.

Statement 4.1. Let $U \in B\left(\mathbb{R}^{d}\right)$ be an orthogonal matrix.

a) if $u$ and $v$ are equivalent Lebesgue measurable on $\mathbb{R}^{d}$ functions, then $u(U(\cdot))$ and $v(U(\cdot))$ are also equivalent Lebesgue measurable functions;

b) if $u \in L_{2}\left(\mathbb{R}^{d}\right)$, then $u(U(\cdot)) \in L_{2}\left(\mathbb{R}^{d}\right)$ and $\|u(U(\cdot))\|_{L_{2}}=\|u\|_{L_{2}}$;

c) if $u, v \in L_{2}\left(\mathbb{R}^{d}\right)$, then $\langle u(U(\cdot)), v(U(\cdot))\rangle=\langle u, v\rangle$.

Proof. a) The measurability of the function $u$ implies the measurability of the function $u(U(\cdot))$. Indeed, if $G$ is an arbitrary open subset of the space $\mathbb{C}$, then the measurability of $u$ implies the Lebesgue measurability of the set $u^{-1}(G) \subset \mathbb{R}^{d}$. Since the family of Lebesgue measurable sets in the space $\mathbb{R}^{d}$ is invariant with respect to the orthogonal transformations of this space, then the set $A^{-1}\left(u^{-1}(G)\right)$ is also measurable.

Let $N \subset \mathbb{R}^{d}$ be a zero measure set, on which equivalent measurable functions $u$ and $v$ differ. Then the functions $u(U(\cdot))$ and $v(U(\cdot))$ are Lebesgue measurable by the above proven facts and differ on the set $U^{-1}(N)$. Since the Lebesgue measure is invariant with respect to ortogonal transformations, the latter set is also of zero measure. Therefore, the functions $u(U(\cdot))$ and $v(U(\cdot))$ are equivalent.

b) Let $u \in L_{2}\left(\mathbb{R}^{d}\right)$. Then there exists a finite integral $\|u\|_{L_{2}}^{2}=\int_{\mathbb{R}^{d}}|u(x)|^{2} d x$. Making an orthogonal change of variables $x=U y$ in this integral and taking into consideration that $|\operatorname{det} U|=1$, we arrive at the needed statement, see [13]. Statement c) can be proved in the same way. The proof is complete.

The proven statement implies that $\mathbf{S}_{\omega}(t)$ is a well-defined unitary operator in $B(\mathcal{H})$.

In order to present further results, we shall need the space $C_{0}\left(\mathbb{R}^{d}\right) \subset \mathcal{H}$ of compactly supported continuous on $\mathbb{R}^{d}$ functions. It is known that this space is everywhere dense in $\mathcal{H}$, see 
[13], and this is why each function in $\mathcal{H}$ is a limit of the functions from $C_{0}\left(\mathbb{R}^{d}\right)$ in the sense of mean-square convergence.

Lemma 4.1. Let $u \in C_{0}\left(\mathbb{R}^{d}\right)$. Then for all $t \geqslant 0$ and $x \in \mathbb{R}^{d}$ the function $u\left(e^{t \mathbf{A}} x\right)$ is a random variable with a finite expectation and $\mathrm{M} u\left(e^{t \mathbf{A}}(\cdot)\right) \in C_{0}\left(\mathbb{R}^{d}\right)$ for all $t \geqslant 0$.

Proof. Let $t \geqslant 0$. For each $x \in \mathbb{R}^{d}$ the function $u\left(e^{t \mathbf{A}} x\right)$ is a random variable as a composition of a continuous function $u$ and a measurable mapping $e^{t \mathbf{A}} x$. Moreover, $u\left(e^{t \mathbf{A}} x\right)$ is bounded on $\Omega$ since $u$ is bounded on $\mathbb{R}^{d}$. Therefore, $\mathrm{M} u\left(e^{t \mathbf{A}} x\right)$ is well-defined for each $x \in \mathbb{R}^{d}$.

For all $x$ and $x_{0}$ in $\mathbb{R}^{d}$ we have

$$
\left|\mathrm{M} u\left(e^{t \mathbf{A}} x\right)-\mathrm{M} u\left(e^{t \mathbf{A}} x_{0}\right)\right|=\left|\mathrm{M}\left(u\left(e^{t \mathbf{A}} x\right)-u\left(e^{t \mathbf{A}} x_{0}\right)\right)\right| \leqslant \mathrm{M}\left|u\left(e^{t \mathbf{A}} x\right)-u\left(e^{t \mathbf{A}} x_{0}\right)\right| .
$$

For each $\omega \in \Omega$ the function $u\left(e^{t \mathbf{A}_{\omega}}(\cdot)\right)$ is continuous on $\mathbb{R}^{d}$ as a composition of continuous mappings $u$ and $e^{t \mathbf{A}_{\omega}}$. Hence, $\left|u\left(e^{t \mathbf{A}} x\right)-u\left(e^{t \mathbf{A}} x_{0}\right)\right|$ converges to zero as $x \rightarrow x_{0}$ pointwise on $\Omega$. At that, $\left|u\left(e^{t \mathbf{A}_{\omega}} x\right)-u\left(e^{t \mathbf{A}_{\omega}} x_{0}\right)\right| \leqslant 2 C$ for all $\omega \in \Omega$ and $x \in \mathbb{R}^{d}$, where the constant $C$ majorizes $u$ on $\mathbb{R}^{d}$. Therefore, by the Lebesgue's dominated convergence theorem, the right hand side in inequality in (4.1) tends to zero as $x \rightarrow x_{0}$, and this is why the same holds for the left hand side in the same inequality. This yields the continuity of the function $\mathrm{M} u\left(e^{t \mathbf{A}}(\cdot)\right)$ on $\mathbb{R}^{d}$.

Finally, if $B$ is a closed ball centered at the zero vector and containing the support of the function $u$, then the supports of the functions $u\left(e^{t \mathbf{A}_{\omega}}(\cdot)\right)$ are contained in $B$ for all $\omega \in \Omega$. Hence, the support of the function $\mathrm{M} u\left(e^{t \mathbf{A}}(\cdot)\right)$ is also contained in $B$. Thus, $\mathrm{M} u\left(e^{t \mathbf{A}}(\cdot)\right)$ is a continuous compactly supported in $\mathbb{R}^{d}$ function, that is, $\mathrm{M} u\left(e^{t \mathbf{A}}(\cdot)\right) \in C_{0}\left(\mathbb{R}^{d}\right)$.

Theorem 4.1. The mapping $\mathbf{S}: \Omega \rightarrow B(\mathcal{H})^{\mathbb{R}_{+}}$with values $\mathbf{S}_{\omega}: \mathbb{R}_{+} \rightarrow B(\mathcal{H})$ defined by the formulae

$$
\mathbf{S}_{\omega}(t) u(\cdot)=u\left(e^{t \mathbf{A}_{\omega}}(\cdot)\right) \quad \forall t \geqslant 0 \quad \forall \omega \in \Omega \quad \forall u \in \mathcal{H},
$$

where $\mathbf{A}: \Omega \rightarrow B\left(\mathbb{R}^{d}\right)$ is a random skew-symmetric matrix, is a random strongly continuous unitary operator semigroup and its averaging $\mathrm{MS}$ is a strongly continuous contractive operatorvalued function with $(\mathrm{MS})(0)=I$. At that, if $u \in C_{0}\left(\mathbb{R}^{d}\right)$, then $(\mathrm{MS})(t) u(\cdot)=\mathrm{M} u\left(e^{t \mathbf{A}}(\cdot)\right) \in$ $C_{0}\left(\mathbb{R}^{d}\right)$ for all $t \geqslant 0$.

Proof. I. Let us check first that $\mathbf{S}_{\omega} \in C_{s}\left(\mathbb{R}_{+}, B(\mathcal{H})\right)$ for all $\omega \in \Omega$. It is obvious that $\mathbf{S}_{\omega}$ is a unitary operator semigroup and hence, according to [10], it is sufficient to confirm that $\left\|\mathbf{S}_{\omega}(t) u-u\right\|_{\mathcal{H}} \stackrel{t \rightarrow 0+}{\longrightarrow} 0$ for all $u$ from a dense subspace $C_{0}\left(\mathbb{R}^{d}\right)$.

We take $u \in C_{0}\left(\mathbb{R}^{d}\right)$. Let $B \subset \mathbb{R}^{d}$ be a closed ball centered at the zero vector and containing the support of the function $u$ and let $|u(x)| \leqslant C$ on $B$. Then for each $t \geqslant 0$ and each $\omega \in \Omega$ the support of the function $u\left(e^{t \mathbf{A}_{\omega}}(\cdot)\right)$ is also contained in $B$ since the ball $B$ is invariant with respect to the orthogonal transformation $e^{t \mathbf{A}_{\omega}}$ mapping bijectively the support of the function $u\left(e^{t \mathbf{A}_{\omega}}(\cdot)\right)$ into the support of the function $u$. This implies that $\left|u\left(e^{t \mathbf{A}_{\omega}} x\right)-u(x)\right|^{2} \leqslant 4 C^{2}$ on $B$ and $\left|u\left(e^{t \mathbf{A}_{\omega}} x\right)-u(x)\right|^{2}=0$ on $\mathbb{R}^{d} \backslash B$. Moreover, by the continuity of the function $u$ and the matrix exponent $e^{t \mathbf{A}_{\omega}}$ as a function of the variable $t$, the function $u\left(e^{t \mathbf{A}_{\omega}}(\cdot)\right)$ converges to $u$ as $t \rightarrow 0+$ pointwise on $\mathbb{R}^{d}$. Then by the Lebesgue theorem on the dominated convergence we get:

$$
\left\|\mathbf{S}_{\omega}(t) u-u\right\|_{\mathcal{H}}^{2}=\int_{B}\left|u\left(e^{t \mathbf{A}_{\omega}} x\right)-u(x)\right|^{2} d x \stackrel{t \rightarrow 0+}{\longrightarrow} 0 \quad \forall u \in C_{0}\left(\mathbb{R}^{d}\right)
$$

and this gives the desired statement. 
II. Now we are going to show that $\mathbf{S}(t)$ is a random operator for each $t \geqslant 0$. In order to do this, it is sufficient to show the measurability of $\langle\mathbf{S}(t) u, v\rangle$ for all $u \in C_{0}\left(\mathbb{R}^{d}\right)$ and $v \in \mathcal{H}$. Moreover, we shall show that $\mathrm{M}\langle\mathbf{S}(t) u, v\rangle \in \mathbb{C}$ is well-defined for such $u$ and $v$ and

$$
\mathrm{M}\langle\mathbf{S}(t) u, v\rangle=\left\langle\mathrm{M} u\left(e^{t \mathbf{A}}(\cdot)\right), v\right\rangle .
$$

Let $t \geqslant 0, u \in C_{0}\left(\mathbb{R}^{d}\right)$ and $v \in \mathcal{H}$. We consider a vector function

$$
f_{t}:(\omega, x) \mapsto e^{t \mathbf{A}_{\omega}} x
$$

on a measurable space $\Omega \times \mathbb{R}^{d}$. The entries of a random matrix $e^{t \mathbf{A}}$ are random variables on $\Omega$ and this is why the components of the vector function $f_{t}$ are measurable on $\Omega \times \mathbb{R}^{d}$ as finite sums of the products of measurable functions. Therefore, $f_{t}$ is a measurable mapping from $\Omega \times \mathbb{R}^{d}$ into $\mathbb{R}^{d}$. At the same time, $u$ is continuous on $\mathbb{R}^{d}$, while $v$ is measurable on $\mathbb{R}^{d}$. Hence, the function

$$
\left(u \circ f_{t}\right) \cdot \bar{v}:(\omega, x) \mapsto u\left(e^{t \mathbf{A}_{\omega}} x\right) \overline{v(x)}
$$

is also measurable on $\Omega \times \mathbb{R}^{d}$.

Since

$$
\mathrm{M}\left(\int_{\mathbb{R}^{d}}\left|u\left(e^{t \mathbf{A}} x\right)\right||v(x)| d x\right) \leqslant \mathrm{M}\left\|\left(u\left(e^{t \mathbf{A}} x\right)\right)\right\|_{\mathcal{H}} \cdot\|v\|_{\mathcal{H}}=\|u\|_{\mathcal{H}}\|v\|_{\mathcal{H}}<\infty
$$

by the Tonelli theorem the function $\left(u \circ f_{t}\right) \cdot \bar{v}$ is integrable on $\Omega \times \mathbb{R}^{d}$. Therefore, by the Fubini theorem, the function

$$
\int_{\mathbb{R}^{d}} u\left(f_{t}(\cdot, x)\right) \overline{v(x)} d x=\langle\mathbf{S}(t) u, v\rangle
$$

is measurable on $\Omega$ and there exists $\mathrm{M}\langle\mathbf{S}(t) u, v\rangle$. Taking into consideration that, by Lemma 4.1. $\mathrm{M} u\left(e^{t \mathbf{A}}(\cdot)\right) \in C_{0}\left(\mathbb{R}^{d}\right)$, again by Fubini theorem we obtain:

$$
\mathrm{M}\langle\mathbf{S}(t) u, v\rangle=\left\langle\mathrm{M} u\left(e^{t \mathbf{A}}(\cdot)\right), v\right\rangle
$$

and this is exactly the needed statement.

It remains to summarize and make final conclusions. According to the facts proven in Items $\mathbf{I}$ and II, $\mathbf{S}$ is a random strongly continuous unitary operator semigroup. Then by Corollary 2.2 , $\mathbf{S}$ possesses averaging MS being a strongly continuous contractive operator-valued function; $(\mathrm{MS})(0)=I$ since $\mathbf{S}_{\omega}(0)=I$ for all $\omega \in \Omega$. And by the facts proved in Item II,

$$
\langle(\mathrm{MS})(t) u, v\rangle=\mathrm{M}\langle\mathbf{S}(t) u, v\rangle=\left\langle\mathrm{M} u\left(e^{t \mathbf{A}}(\cdot)\right), v\right\rangle \quad \forall t \geqslant 0 \quad \forall u \in C_{0}\left(\mathbb{R}^{d}\right) \quad \forall v \in \mathcal{H} .
$$

Since this identity holds for all $v \in \mathcal{H}$, then

$$
(\mathrm{MS})(t) u=\mathrm{M} u\left(e^{t \mathbf{A}}(\cdot)\right) \quad \forall t \geqslant 0 \quad \forall u \in C_{0}\left(\mathbb{R}^{d}\right),
$$

this completes the proof of Theorem 4.1 .

Since the function $t \mapsto \sqrt{t}$ is continuous on $\mathbb{R}_{+}$, the strong continuity of $\mathbf{S}_{\omega}$ implies a strong continuity of $\mathbf{T}_{\omega}: t \mapsto \mathbf{S}_{\omega}(\sqrt{t})$. Then by Theorem 4.1 we obtain the following corollary.

Corollary 4.1. The mapping $\mathbf{T}: \Omega \rightarrow B(\mathcal{H})^{\mathbb{R}_{+}}$with values $\mathbf{T}_{\omega}: \mathbb{R}_{+} \rightarrow B(\mathcal{H})$ defined by the formulae

$$
\mathbf{T}_{\omega}(t) u(\cdot)=u\left(e^{\sqrt{t} \mathbf{A}_{\omega}}(\cdot)\right) \quad \forall t \geqslant 0 \quad \forall \omega \in \Omega \quad \forall u \in \mathcal{H}
$$

where $\mathbf{A}: \Omega \rightarrow B\left(\mathbb{R}^{d}\right)$ is a random skew-symmetric matrix is a random strongly continuous unitary operator-valued function and its averaging MT is a strongly continuous contractive 
operator-valued function with $(\mathrm{MT})(0)=I$. At that, if $u \in C_{0}\left(\mathbb{R}^{d}\right)$, then $(\mathrm{MT})(t) u(\cdot)=$ $\mathrm{M} u\left(e^{\sqrt{t} \mathbf{A}}(\cdot)\right) \in C_{0}\left(\mathbb{R}^{d}\right)$ for all $t \geqslant 0$.

4.2. Derivative of averaging at zero. Now we are going to study a strong derivative of the averagings MS and MT at zero. In order to do this, we shall make use of the space $C_{0}^{k}\left(\mathbb{R}^{d}\right) \subset \mathcal{H}$ of $k$ times continuously differentiable compactly supported on $\mathbb{R}^{d}$ functions. As $C_{0}\left(\mathbb{R}^{d}\right)$, this space is everywhere dense in $\mathcal{H}$, see [13].

Theorem 4.2. Let MS be the operator-valued function from Theorem 4.1 and let MA $\in$ $B\left(\mathbb{R}^{d}\right)$ be well-defined. Then for each $u \in C_{0}^{1}\left(\mathbb{R}^{d}\right)$ there exists

$$
(\mathrm{MS})^{\prime}(0) u=\lim _{t \rightarrow 0+} \frac{(\mathrm{MS})(t) u-u}{t}=(\nabla u,(\mathrm{MA}) x),
$$

where the limit is treated in the sense of the convergence in $\mathcal{H}$.

Proof. Let as above $B \subset \mathbb{R}^{d}$ be a closed ball centered at the zero vector and containing the support of the function $u$. Since this function is continuously differentiable and compactly supported, there exists $M_{1}=\max _{x \in B}|\nabla u(x)|$. By the Taylor formula, for all points $x$ and $y$ in the ball $B$ the identity

$$
u(y)=u(x)+(\nabla u(x), y-x)+r_{1}(x, y) \cdot|y-x|
$$

holds, where $r_{1}(x, y)$ satisfies the conditions

$$
\begin{array}{ll}
\left|r_{1}(x, y)\right| \leqslant 2 M_{1} \quad \forall x \in B \quad \forall y \in B, \\
\lim _{y \rightarrow x} r_{1}(x, y)=0 \quad \forall x \in B .
\end{array}
$$

Substituting $y=e^{t \mathbf{A}_{\omega}} x \in B$ into identity 4.2 , for each $\omega \in \Omega$ and $t \geqslant 0$ we obtain:

$$
u\left(e^{t \mathbf{A}_{\omega}} x\right)-u(x)=\left(\nabla u(x), e^{t \mathbf{A}_{\omega}} x-x\right)+r_{1}\left(x, e^{t \mathbf{A}_{\omega}} x\right) \cdot\left|e^{t \mathbf{A}_{\omega}} x-x\right| .
$$

According to Theorem 4.1, there exists $\mathrm{M} u\left(e^{t \mathbf{A}}(\cdot)\right)=(\mathrm{MS})(t) u \in C_{0}\left(\mathbb{R}^{d}\right)$ for all $t \geqslant 0$. Moreover, a random matrix $e^{t \mathbf{A}}$ also possesses an averaging $\mathrm{M}\left(e^{t \mathbf{A}}\right)$. Then the remainder term in the right hand side of identity (4.5) also possesses an averaging, which for each $t \geqslant 0$ is a function from the space $C_{0}\left(\mathbb{R}^{d}\right)$. Dividing identity 4.5 by $t$ and passing to the expectation, we obtain:

$$
\frac{(\mathrm{MS})(t) u(x)-u(x)}{t}=\left(\nabla u(x), \frac{\mathrm{M}\left(e^{t \mathbf{A}}\right) x-x}{t}\right)+\mathrm{M}\left(r_{1}\left(x, e^{t \mathbf{A}} x\right) \cdot\left|\frac{e^{t \mathbf{A}} x-x}{t}\right|\right) .
$$

Let us show that the last term in the right hand side of identity 4.6 tends to zero in $\mathcal{H}$ as $t \rightarrow 0+$. Taking into consideration (4.3) and the estimate $\left\|e^{t \mathbf{A}}-I\right\| \leqslant t\|\mathbf{A}\|$ proved in Statement 3.1, we obtain:

$$
\left|r_{1}\left(x, e^{t \mathbf{A}} x\right)\right| \cdot\left|\frac{e^{t \mathbf{A}} x-x}{t}\right| \leqslant 2 M_{1} r_{B}\|\mathbf{A}\|,
$$

where $r_{B}$ is the radius of the ball $B$. The existence of MA implies the existence $\mathbf{M}\|\mathbf{A}\|$ and this is why for each $x \in B$ the left hand side of inequality (4.7) is majorized by an integrable random variable. Moreover, by 4.4 the function $r_{1}\left(x, e^{t \mathbf{A}} x\right)$ tends to zero as $t \rightarrow 0+$ pointwise on $\Omega$ for each $x \in B$, while $\frac{e^{t \mathbf{A}} x-x}{t}$ tends to $\mathbf{A} x$. This is why the left hand side of inequality (4.7) tends to zero as $t \rightarrow 0+$ point-wise on $\Omega$ for each $x \in B$. Hence, by the Lebesgue's dominated convergence theorem, the last term in the right hand side of identity 4.6 tends to zero as $t \rightarrow 0+$ point-wise on $B$. At that, it follows from estimate 4.7 that

$$
\left|\mathrm{M}\left(r_{1}\left(x, e^{t \mathbf{A}} x\right) \cdot\left|\frac{e^{t \mathbf{A}} x-x}{t}\right|\right)\right| \leqslant 2 M_{1} r_{B} \mathrm{M}\|\mathbf{A}\|<\infty,
$$


and this is why by the Lebesgue theorem on majorized convergence the last term in the right hand side of identity (4.6) tends to zero as $t \rightarrow 0+$ also in the space $\mathcal{H}$; outside the ball $B$ this term is identically zero.

It remains to find the limit of the first term in the right hand side of (4.6). In order to do this, we note that by Theorem 3.1

$$
\begin{aligned}
\left|\left(\nabla u(x), \frac{\mathrm{M}\left(e^{t \mathbf{A}}\right) x-x}{t}\right)-(\nabla u(x),(\mathrm{MA}) x)\right| & =\left|\left(\nabla u(x), \frac{\mathrm{M}\left(e^{t \mathbf{A}}\right)-I-t \mathrm{MA}}{t} x\right)\right| \\
& \leqslant|\nabla u| \cdot|x| \cdot\left\|\frac{\mathrm{M}\left(e^{t \mathbf{A}}\right)-I-t \mathrm{MA}}{t}\right\| \\
& \leqslant M_{1} r_{B}\left\|\frac{\mathrm{M}\left(e^{t \mathbf{A}}\right)-I-t \mathrm{MA}}{t}\right\| \stackrel{t \rightarrow 0+}{\rightarrow} 0,
\end{aligned}
$$

which implies the uniform on $B$ convergence of this term to $(\nabla u,(\mathrm{MA}) x)$ and hence, the same convergence in $\mathcal{H}$. The proof is complete.

Theorem 4.3. Let $\mathrm{MT}$ be an operator-valued function in Corollary 4.1 and let there exist $\mathrm{M}\left(\mathbf{A}^{2}\right) \in B\left(\mathbb{R}^{d}\right)$ and $\mathrm{MA}=0$. Then for each $u \in C_{0}^{2}\left(\mathbb{R}^{d}\right)$ there exists

$$
(\mathrm{MT})^{\prime}(0) u=\lim _{t \rightarrow 0+} \frac{(\mathrm{MT})(t) u-u}{t}=\frac{1}{2}\left(\nabla u, \mathrm{M}\left(\mathbf{A}^{2}\right) x\right)-\frac{1}{2}\left(x, \mathrm{M}\left(\mathbf{A} H_{u} \mathbf{A}\right) x\right),
$$

wherew $H_{u}$ is the matrix of the second derivatives of the functions $u$ (Hessian matrix). The limit is treated in the sense of the convergence in $\mathcal{H}$.

Proof. Similar to the proof of Theorem 4.2 , we consider a closed ball $B \subset \mathbb{R}^{d}$ centered at the zero vector and containing the support of the function $u$. Let $M_{1}=\max _{x \in B}|\nabla u|, M_{2}=\max _{x \in B}\left\|H_{u}(x)\right\|$. We expand $u$ in the ball $B$ by the Taylor formula

$$
u(y)=u(x)+(\nabla u(x), y-x)+\frac{1}{2}\left(y-x, H_{u}(x)(y-x)\right)+r_{2}(x, y) \cdot|y-x|^{2},
$$

where $r_{2}(x, y)$ satisfies the conditions

$$
\begin{aligned}
& \left|r_{2}(x, y)\right| \leqslant M_{2} \quad \forall x \in B \quad \forall y \in B, \\
& \lim _{y \rightarrow x} r_{2}(x, y)=0 \quad \forall x \in B .
\end{aligned}
$$

Substituting $y=e^{\sqrt{t} \mathbf{A}_{\omega}} x \in B$ into identity $(4.9)$, for each $\omega \in \Omega$ and $t \geqslant 0$ we obtain:

$$
\begin{aligned}
u\left(e^{\sqrt{t} \mathbf{A}_{\omega}} x\right)-u(x)= & \left(\nabla u(x), e^{\sqrt{t} \mathbf{A}_{\omega}} x-x\right)+\frac{1}{2}\left(e^{\sqrt{t} \mathbf{A}_{\omega}} x-x, H_{u}(x)\left(e^{\sqrt{t} \mathbf{A}_{\omega}} x-x\right)\right) \\
& +r_{2}\left(x, e^{\sqrt{t} \mathbf{A}_{\omega}} x\right) \cdot\left|e^{\sqrt{t} \mathbf{A}_{\omega}} x-x\right|^{2} .
\end{aligned}
$$

According to Corollary 4.1. there exists $\mathrm{M} u\left(e^{\sqrt{t} \mathbf{A}}(\cdot)\right)=(\mathrm{MT})(t) u \in C_{0}\left(\mathbb{R}^{d}\right)$ as well as $\mathrm{M}\left(e^{\sqrt{t} \mathbf{A}}\right) \in B\left(\mathbb{R}^{d}\right)$ for all $t \geqslant 0$. Moreover, since by Statement 3.1

$$
\left|\left(e^{\sqrt{t} \mathbf{A}} x-x, H_{u}(x)\left(e^{\sqrt{t} \mathbf{A}} x-x\right)\right)\right| \leqslant\left\|H_{u}(x)\right\| \cdot|x|^{2} \cdot\left\|e^{\sqrt{t} \mathbf{A}}-I\right\|^{2} \leqslant t\left\|H_{u}(x)\right\| \cdot|x|^{2} \cdot\|\mathbf{A}\|^{2}
$$

and the existence of $\mathrm{M}\left(\mathbf{A}^{2}\right)$ implies the integrability of the random variable $\|\mathbf{A}\|^{2}$ on $\Omega$, then there exists an averaging of the second term in the right hand side of identity (4.12); at that, since this term is a sum of products of the coordinates of the vector $x$ and of the entries of the Hessian matrix $H_{u}(x)$, being continuous functions, with the entries of the random matrix $e^{\sqrt{t} \mathbf{A}}-I$, then after averaging, for each $t \geqslant 0$, it is a compactly supported continuous in $x$ function. The above said facts imply that there exists an averaging of the remainder term in 
identity 4.12); for each $t \geqslant 0$ it is a function from the space $C_{0}\left(\mathbb{R}^{d}\right)$. We divide identity 4.12 ) by $t$, pass to expectation and, taking into consideration that $\left(e^{\sqrt{t} \mathbf{A}}-I\right)^{*}=e^{-\sqrt{t} \mathbf{A}}-I$ by the orthogonality of the matrix $e^{\sqrt{t} \mathbf{A}}$, we obtain:

$$
\begin{aligned}
\frac{(\mathrm{MT})(t) u(x)-u(x)}{t}= & \left(\nabla u(x), \frac{\mathrm{M}\left(e^{\sqrt{t} \mathbf{A}}\right) x-x}{t}\right) \\
& +\frac{1}{2}\left(x, \frac{\mathrm{M}\left(\left(e^{-\sqrt{t} \mathbf{A}}-I\right) H_{u}(x)\left(e^{\sqrt{t} \mathbf{A}}-I\right)\right) x}{t}\right) \\
& +\mathrm{M}\left(r_{2}\left(x, e^{\sqrt{t} \mathbf{A}} x\right) \cdot\left|\frac{e^{\sqrt{t} \mathbf{A}} x-x}{\sqrt{t}}\right|^{2}\right) .
\end{aligned}
$$

We are going to find the limit of each term in the right hand side of $(4.13)$ as $t \rightarrow 0+$. Let $r_{B}$ be the radius of the ball $B$. By Theorem 3.1 and the condition $\mathrm{MA}=0$, for the first term we have

$$
\begin{aligned}
\left|\left(\nabla u(x), \frac{\mathrm{M}\left(e^{\sqrt{t} \mathbf{A}}\right) x-x}{t}\right)-\frac{1}{2}\left(\nabla u(x), \mathrm{M}\left(\mathbf{A}^{2}\right) x\right)\right| & =\left|\left(\nabla u(x), \frac{\mathrm{M}\left(e^{\sqrt{t} \mathbf{A}}\right)-I-\frac{t}{2} \mathrm{M}\left(\mathbf{A}^{2}\right)}{t} x\right)\right| \\
& \leqslant|\nabla u(x)| \cdot|x| \cdot\left\|\frac{\mathrm{M}\left(e^{\sqrt{t} \mathbf{A}}\right)-I-\frac{t}{2} \mathrm{M}\left(\mathbf{A}^{2}\right)}{t}\right\| \\
& \leqslant M_{1} r_{B}\left\|\frac{\mathrm{M}\left(e^{\sqrt{t} \mathbf{A}}\right)-I-\frac{t}{2} \mathrm{M}\left(\mathbf{A}^{2}\right)}{t}\right\| \stackrel{t \rightarrow 0+}{\longrightarrow} 0,
\end{aligned}
$$

which implies the uniform on $B$ convergence (and hence the convergence in $\mathcal{H}$ ) of this term to $\frac{1}{2}\left(\nabla u(x), \mathrm{M}\left(\mathbf{A}^{2}\right) x\right)$.

For the second term we first note that in view of Statement 3.1

$$
\begin{aligned}
\frac{1}{t} & \left\|\left(e^{-\sqrt{t} \mathbf{A}}-I\right) H_{u}(x)\left(e^{\sqrt{t} \mathbf{A}}-I\right)+t \mathbf{A} H_{u}(x) \mathbf{A}\right\| \\
& \leqslant \frac{1}{t}\left\|\left(e^{-\sqrt{t} \mathbf{A}}-I\right) H_{u}(x)\left(e^{\sqrt{t} \mathbf{A}}-I-\sqrt{t} \mathbf{A}\right)\right\|+\frac{1}{t}\left\|\left(e^{-\sqrt{t} \mathbf{A}}-I+\sqrt{t} \mathbf{A}\right) H_{u}(x) \sqrt{t} \mathbf{A}\right\| \\
& \leqslant \frac{1}{t} \cdot \sqrt{t}\|\mathbf{A}\| \cdot\left\|H_{u}(x)\right\| \cdot 2 \sqrt{t}\|\mathbf{A}\|+\frac{1}{t} \cdot 2 \sqrt{t}\|\mathbf{A}\| \cdot\left\|H_{u}(x)\right\| \cdot \sqrt{t}\|\mathbf{A}\| \leqslant 4 M_{2} \cdot\left\|\mathbf{A}^{2}\right\| .
\end{aligned}
$$

Thus, the left hand side of inequality (4.14) for each $x \in B$ is majorized by an integrable random variable $4 M_{2}\left\|\mathbf{A}^{2}\right\|$. On the other hand, by the same statement,

$$
\begin{aligned}
\frac{1}{t} & \left\|\left(e^{-\sqrt{t} \mathbf{A}}-I\right) H_{u}(x)\left(e^{\sqrt{t} \mathbf{A}}-I\right)+t \mathbf{A} H_{u}(x) \mathbf{A}\right\| \\
& \leqslant \frac{1}{t}\left\|\left(e^{-\sqrt{t} \mathbf{A}}-I\right) H_{u}(x)\left(e^{\sqrt{t} \mathbf{A}}-I-\sqrt{t} \mathbf{A}\right)\right\|+\frac{1}{t}\left\|\left(e^{-\sqrt{t} \mathbf{A}}-I+\sqrt{t} \mathbf{A}\right) H_{u}(x) \sqrt{t} \mathbf{A}\right\| \\
& \leqslant \frac{1}{t} \cdot \sqrt{t}\|\mathbf{A}\| \cdot\left\|H_{u}(x)\right\| \cdot \frac{t}{2}\left\|\mathbf{A}^{2}\right\|+\frac{1}{t} \cdot \frac{t}{2}\left\|\mathbf{A}^{2}\right\| \cdot\left\|H_{u}(x)\right\| \cdot \sqrt{t}\|\mathbf{A}\| \\
& \leqslant \sqrt{t} M_{2} \cdot\left\|\mathbf{A}^{3}\right\| \stackrel{t \rightarrow 0+}{\longrightarrow} 0,
\end{aligned}
$$

i.e., for each $x \in B$ the left hand side of inequality 4.15 converges to zero as $t \rightarrow 0+$ point-wise in $\Omega$. Then by Statement 2.3

$$
\frac{1}{t}\left\|\mathrm{M}\left(\left(e^{-\sqrt{t} \mathbf{A}}-I\right) H_{u}(x)\left(e^{\sqrt{t} \mathbf{A}}-I\right)\right)+t \mathrm{M}\left(\mathbf{A} H_{u}(x) \mathbf{A}\right)\right\| \stackrel{t \rightarrow 0+}{\longrightarrow} 0 \quad \forall x \in B .
$$


Employing now estimate (4.14), by Statement 2.2, on $B$ we obtain:

$$
\begin{aligned}
& \left|\left(x, \frac{\mathrm{M}\left(\left(e^{-\sqrt{t} \mathbf{A}}-I\right) H_{u}(x)\left(e^{\sqrt{t} \mathbf{A}}-I\right)\right) x}{t}\right)+\left(x, \mathrm{M}\left(\mathbf{A} H_{u}(x) \mathbf{A}\right) x\right)\right| \\
& \quad=\left|\left(x, \frac{1}{t} \mathrm{M}\left(\left(e^{-\sqrt{t} \mathbf{A}}-I\right) H_{u}(x)\left(e^{\sqrt{t} \mathbf{A}}-I\right)+t \mathbf{A} H_{u}(x) \mathbf{A}\right) x\right)\right| \\
& \leqslant|x|^{2} \cdot\left\|\frac{1}{t} \mathrm{M}\left(\left(e^{-\sqrt{t} \mathbf{A}}-I\right) H_{u}(x)\left(e^{\sqrt{t} \mathbf{A}}-I\right)+t \mathbf{A} H_{u}(x) \mathbf{A}\right)\right\| \leqslant 4 M_{2} r_{B}^{2} \mathrm{M}\left\|\mathbf{A}^{2}\right\|<\infty,
\end{aligned}
$$

and this is why the Lebesgue's dominated convergence theorem and 4.16$)$ imply the convergence of the second term in the right hand side of identity 4.13 to $-\frac{1}{2}\left(x, \mathrm{M}\left(\mathbf{A} H_{u} \mathbf{A}\right) x\right)$ in $\mathcal{H}$.

Taking into consideration 4.10 and the estimate $\left\|e^{t \mathbf{A}}-I\right\| \leqslant t\|\mathbf{A}\|$ proved in Statement 3.1 . we obtain:

$$
\left|r_{2}\left(x, e^{\sqrt{t} \mathbf{A}} x\right)\right| \cdot\left|\frac{e^{\sqrt{t} \mathbf{A}} x-x}{\sqrt{t}}\right|^{2} \leqslant M_{2} r_{B}^{2}\left\|\mathbf{A}^{2}\right\| .
$$

Since the random variable $\left\|\mathbf{A}^{2}\right\|$ is integrable, for each $x \in B$ the left hand side in (4.17) is majorized by an integrable function. Moreover, by 4.11), for each $x \in B$, the function $r_{2}\left(x, e^{\sqrt{t} \mathbf{A}} x\right)$ tends to zero as $t \rightarrow 0+$ pointwise on $\Omega$, while $\frac{e^{\sqrt{t} \mathbf{A}} x-x}{\sqrt{t}}$ tends to $\mathbf{A} x$. This is why the left hand side in (4.17) tends to zero. Hence, by the Lebesgue's dominated convergence theorem the last term in the right hand side of identity $(4.13)$ tends to zero as $t \rightarrow 0+$ pointwise on $B$. At that, it follows from estimate 4.17 that

$$
\left|\mathrm{M}\left(r_{2}\left(x, e^{\sqrt{t} \mathbf{A}} x\right) \cdot\left|\frac{e^{\sqrt{t} \mathbf{A}} x-x}{\sqrt{t}}\right|^{2}\right)\right| \leqslant M_{2} r_{B}^{2} \mathrm{M}\left\|\mathbf{A}^{2}\right\|<\infty
$$

on $B$. This is why by the Lebesgue's dominated convergence theorem, the last term in the right hand side of identity 4.13 tends to zero as $t \rightarrow 0+$ in the space $\mathcal{H}$. The proof is complete.

4.3. Divergence form of derivative. Operator 4.8 can be written in a divergence form if for each $x \in \mathbb{R}^{d}$ and each $\omega \in \Omega$ we introduce the tensor product $\mathbf{A}_{\omega} x \otimes \mathbf{A}_{\omega} x$, which is considered as a bilinear form on $\mathbb{C}^{d}$ acting on the pair of the vectors $\xi, \eta$ by the rule

$$
\left(\mathbf{A}_{\omega} x \otimes \mathbf{A}_{\omega} x\right)(\xi, \eta)=\left(\mathbf{A}_{\omega} x, \xi\right)\left(\mathbf{A}_{\omega} x, \eta\right) .
$$

The averaging of this tensor is introduced component-wise as in the case of the operators, that is, by $\mathrm{M}(\mathbf{A} x \otimes \mathbf{A} x)$ we mean the tensor acting on a pair of vectors $\xi, \eta$ by the rule

$$
\mathrm{M}(\mathbf{A} x \otimes \mathbf{A} x)(\xi, \eta)=\mathrm{M}((\mathbf{A} x, \xi)(\mathbf{A} x, \eta)) .
$$

The existence of the averaging of this tensor is implied by the existence of a finite $\mathrm{M}\|\mathbf{A}\|^{2}$, which is true by the assumptions of Theorem 4.3 .

In what follows, it will be convenient to regard the tensor $\mathbf{A} x \otimes \mathbf{A} x$ as an operator from $\mathbb{C}^{d}$ into $\mathbb{C}^{d}$, which maps each vector $\xi$ into the vector

$$
(\mathbf{A} x \otimes \mathbf{A} x) \xi=(\mathbf{A} x, \xi) \mathbf{A} x .
$$

We note that then the identity

$$
\begin{aligned}
\nabla \cdot(\mathbf{A} x \otimes \mathbf{A} x) \nabla u & =\nabla \cdot((\mathbf{A} x, \nabla u) \mathbf{A} x)=\left(\mathbf{A}^{2} x, \nabla u\right)+\left(\mathbf{A} x, H_{u} \mathbf{A} x\right)+(\mathbf{A} x, \nabla u) \operatorname{tr} \mathbf{A} \\
& =\left(\nabla u, \mathbf{A}^{2} x\right)-\left(x, \mathbf{A} H_{u} \mathbf{A} x\right)
\end{aligned}
$$


holds for an arbitrary function $u \in C_{0}^{2}\left(\mathbb{R}^{d}\right)$; here in the latter identity we have taken into consideration that $\operatorname{tr} \mathbf{A}=0$ and $(\mathbf{A} x, y)=-(x, \mathbf{A} y)$ by the skew-symmetricity of $\mathbf{A}$. Applying now averaging and employing the linearity of the operators $\mathbf{A}, \nabla$ and of the scalar product and comparing with 4.8 , we conclude that

$$
(\mathrm{MT})^{\prime}(0) u=\frac{1}{2} \nabla \cdot(\mathrm{M}(\mathbf{A} x \otimes \mathbf{A} x) \nabla u) \quad \forall u \in C_{0}^{2}\left(\mathbb{R}^{d}\right) .
$$

4.4. Study of derivative in $L_{2}\left(\mathbb{R}^{d}\right)$. For the sake of brevity we denote operator 4.18 by $-\frac{1}{2} L$. A sesquilinear form associated with the operator $L$ reads as

$$
\begin{aligned}
\langle L u, v\rangle=-\int_{\mathbb{R}^{d}} \nabla \cdot(\mathrm{M}(\mathbf{A} x \otimes \mathbf{A} x) \nabla u) \bar{v} d x & =\int_{\mathbb{R}^{d}}(\mathrm{M}(\mathbf{A} x \otimes \mathbf{A} x) \nabla u, \overline{\nabla v}) d x \\
& =\int_{\mathbb{R}^{d}} \mathrm{M}((\mathbf{A} x, \nabla u)(\mathbf{A} x, \overline{\nabla v})) d x,
\end{aligned}
$$

where we have integrated by parts and employed that the functions $u$ and $v$ are compactly supported. In particular,

$$
\langle L u, u\rangle=\int_{\mathbb{R}^{d}} \mathrm{M}|(\mathbf{A} x, \nabla u)|^{2} d x \geqslant 0,
$$

that is, the operator $L$ is non-negative on $C_{0}^{2}\left(\mathbb{R}^{d}\right)$ and therefore, it is symmetric. However, it is not elliptic. Indeed, let $u(x)=v\left(|x|^{2}\right)$, where $v \in C_{0}^{\infty}(\mathbb{R})$. Then $u \in C_{0}^{\infty}\left(\mathbb{R}^{d}\right)$ and $\nabla u=2 x v^{\prime}\left(|x|^{2}\right)$, and this is why

$$
(\mathbf{A} x, \nabla u)=2 v^{\prime}\left(|x|^{2}\right)(\mathbf{A} x, x)=0 \quad \forall \omega \in \Omega \quad \forall x \in \mathbb{R}^{d}
$$

by the skew-symmetricity of $\mathbf{A}$. Hence, on such functions we have $\langle L u, u\rangle=0$.

The matter is that the operator $L$ degenerates along the radial direction, what can be also seen from the definition of this operator as a derivative of MT: on functions $u$ depending only on $|x|$ we have $(\mathrm{MT})(t) u=u$ for each $t$. This arguing leads us to the conclusion that it is natural to consider the operator $L$ in polar coordinates, in which it should contain no derivatives with respect to $|x|$. Let us check this by straightforward calculations.

We let $x=r \sigma$ with $r=|x|>0$, and $\sigma=\frac{x}{r} \in \mathbb{S}^{d-1}$, where $\mathbb{S}^{d-1}$ is a $(d-1)$-dimensional unit sphere in the space $\mathbb{R}^{d}$. Then $\nabla=\sigma \frac{\partial}{\partial r}+\frac{1}{r} \nabla_{\mathbb{S}^{d-1}}$, where $\nabla_{\mathbb{S}^{d-1}}$ is the gradient on $\mathbb{S}^{d-1}$. Substituting this into 4.18 and taking into consideration that $(\mathbf{A} \sigma, \sigma)=0$, we successively obtain:

$$
\begin{aligned}
\mathrm{M}(\mathbf{A} x \otimes \mathbf{A} x) \nabla u & =r^{2} \mathrm{M}(\mathbf{A} \sigma \otimes \mathbf{A} \sigma)\left(\sigma u_{r}^{\prime}+\frac{1}{r} \nabla_{\mathbb{S}^{d-1}} u\right) \\
& =r^{2} u_{r}^{\prime} \mathrm{M}((\mathbf{A} \sigma, \sigma) \mathbf{A} \sigma)+r \mathrm{M}(\mathbf{A} \sigma \otimes \mathbf{A} \sigma) \nabla_{\mathbb{S}^{d-1}} u \\
& =r \mathrm{M}(\mathbf{A} \sigma \otimes \mathbf{A} \sigma) \nabla_{\mathbb{S}^{d-1}} u \\
\nabla \cdot(\mathrm{M}(\mathbf{A} x \otimes \mathbf{A} x) \nabla u) & =\left(\sigma \frac{\partial}{\partial r}+\frac{1}{r} \nabla_{\mathbb{S}^{d-1}}\right) \cdot\left(r \mathrm{M}(\mathbf{A} \sigma \otimes \mathbf{A} \sigma) \nabla_{\mathbb{S}^{d-1}} u\right) \\
& =\sigma \cdot \mathrm{M}(\mathbf{A} \sigma \otimes \mathbf{A} \sigma)\left(r \nabla_{\mathbb{S}^{d-1}} u\right)_{r}^{\prime}+\nabla_{\mathbb{S}^{d-1}} \cdot\left(\mathrm{M}(\mathbf{A} \sigma \otimes \mathbf{A} \sigma) \nabla_{\mathbb{S}^{d-1}} u\right) \\
& =\mathrm{M}\left((\mathbf{A} \sigma, \sigma)\left(\mathbf{A} \sigma,\left(r \nabla_{\mathbb{S}^{d-1}} u\right)_{r}^{\prime}\right)\right)+\nabla_{\mathbb{S}^{d-1}} \cdot\left(\mathrm{M}(\mathbf{A} \sigma \otimes \mathbf{A} \sigma) \nabla_{\mathbb{S}^{d-1}} u\right) \\
& =\nabla_{\mathbb{S}^{d-1}} \cdot\left(\mathrm{M}(\mathbf{A} \sigma \otimes \mathbf{A} \sigma) \nabla_{\mathbb{S}^{d-1}} u\right) .
\end{aligned}
$$

Thus,

$$
L=-\nabla_{\mathbb{S}^{d-1}} \cdot\left(\mathrm{M}(\mathbf{A} \sigma \otimes \mathbf{A} \sigma) \nabla_{\mathbb{S}^{d-1}} u\right)
$$


4.5. Study of derivative in $L_{2}\left(\mathbb{S}^{d-1}\right)$. We note that for each point $\sigma \in \mathbb{S}^{d-1}$ the vector $\mathbf{A} \sigma$ is orthogonal to $\sigma$ and this is why we can suppose that $\mathbf{A} \sigma$ belongs to a complexified tangent space $T_{\sigma} \mathbb{S}^{d-1}$ to the sphere $\mathbb{S}^{d-1}$ at the point $\sigma$. Therefore, we can suppose that the tensor $\mathrm{M}(\mathbf{A} \sigma \otimes \mathbf{A} \sigma)$ acts in $T_{\sigma} \mathbb{S}^{d-1}$. At that, the spherical gradient $\nabla_{\mathbb{S}^{d-1}}$ is a vector operator with values in $T_{\sigma} \mathbb{S}^{d-1}$ at each point $\sigma \in \mathbb{S}^{d-1}$. Thus, the operator $L$ represented by formula 4.19 is a well-defined operator in the space $L_{2}\left(\mathbb{S}^{d-1}\right)$ with the domain $C^{2}\left(\mathbb{S}^{d-1}\right)$.

Theorem 4.4. Let a random matrix A satisfies the assumptions of Theorem 4.3 and there exists a constant $\gamma>0$ such that

$$
\mathrm{M}|(\mathbf{A} \sigma, \xi)|^{2} \geqslant \gamma|\xi|^{2} \quad \forall \sigma \in \mathbb{S}^{d-1} \quad \forall \xi \in T_{\sigma} \mathbb{S}^{d-1} .
$$

Then the operator $L$ defined on the space $L_{2}\left(\mathbb{S}^{d-1}\right)$ by formula (4.19) with the domain $C^{2}\left(\mathbb{S}^{d-1}\right)$ is essentially self-adjoint, while the domain of its closure coincides with the Sobolev space $W_{2}^{2}\left(\mathbb{S}^{d-1}\right)$.

Proof. In the same way as above we establish the non-negativity and symmetricity of the operator $L$, while condition 4.20 makes operator $L$ elliptic. Moreover, the coefficients of the form $\mathrm{M}(\mathbf{A} \sigma \otimes \mathbf{A} \sigma)$ are infinitely differentiable in each local coordinate system and are bounded by the number $M\|\mathbf{A}\|^{2}$. The sphere $\mathbb{S}^{d-1}$ is a compact Riemannian manifold. Thus, $L$ is a symmetric elliptic operator with bounded infinitely differentiable coefficients defined on the space $L_{2}\left(\mathbb{S}^{d-1}\right)$ with the domain $C^{2}\left(\mathbb{S}^{d-1}\right)$. According to [14], the statement of the theorem holds true for such operator. The proof is complete.

Theorem 4.4 is a key result of this study: it provides conditions under which the derivative of the averaging of a random operator-valued function $\mathbf{T}(t)=\mathbf{S}(\sqrt{t})$ is an essentially self-adjoint operator, and therefore, it is a generator of some strongly continuous operator semigroup. It implies a final result of the paper.

Corollary 4.2. Let a random matrix A satisfies the assumptions of Theorems 4.3 and 4.4. Then the operator-valued function MT is Chernoff equivalent to a contractive strongly continuous semigroup generated by the closure of the operator $-\frac{L}{2}$, where $L$ is defined by formula (4.19).

Proof. By Theorem 4.4, the operator $L$ is non-negative and essentially self-adjoint. Then the operator $-\frac{L}{2}=(\mathrm{MT})^{\prime}(0)$ is non-positive essentially self-adjoint and this is why its closure generates a contractive strongly continuous semigroup. It remains to apply the Chernoff theorem in order to complete the proof.

\section{ACKNOWLEDGMENTS}

The author is grateful to V.Zh. Sakbaev for fruitful discussion of the problems considered in the work.

\section{BIBLIOGRAPHY}

1. H. Furstenberg, H. Kesten. Products of random matrices // Ann. Math. Statist. 31:2, 457-469 (1960).

2. H. Furstenberg. Noncommuting random products // Trans. Amer. Math. Soc. 108:3, 377-428 (1963).

3. V.N. Tutubalin. On limit theorems for products of random matrices // Teor. Veroyat. Prim. 10:1, 19-32 (1965). [Theory Probab. Appl. 10:1, 15-27 (1965).]

4. V.I. Oseledets. A multiplicative ergodic theorem. Characteristic Ljapunov, exponents of dynamical systems // Trudy Moskov. Matem. Obsh. 19, 179-210 (1968). [Trans. Moscow Math. Soc. 19, 197-231 (1968).] 
5. A.V. Skorokhod. Operator stochastic differential equations and stochastic semigroups // Uspekhi Matem. Nauk. 37:6, 157-183 (1982). [Russ. Math. Surv. 37:6, 177-204 (1982).]

6. V.Zh. Sakbaev. On the law of large numbers for compositions of independent random semigroups // Izv. VUZov. Matem. 10, 86-91 (2016). [Russ. Math. (Iz. VUZ). 60:10, 72-76 (2016).]

7. V.Zh. Sakbaev. Averaging of random flows of linear and nonlinear maps // J. Phys. Conf. Ser. 990, 012012 (2018).

8. Yu.N. Orlov, V.Zh. Sakbaev, O.G. Smolyanov. Feynman formulas and the law of large numbers for random one-parameter semigroups // Trudy MIAN. 306, 210-226 (2019). [Proc. Steklov Inst. Math. 306, 196-211 (2019).]

9. K.Yu. Zamana, V.Zh. Sakbaev, O.G. Smolyanov. Stochastic processes on the group of orthogonal matrices and evolution equations describing them // Zhurn. Vychisl. Matem. Matem. Fiz. 60:10, 1741-1756 (2020). [Comput. Math. Math. Phys. 60:10, 1686-1700 (2020).]

10. K.J. Engel, R. Nagel. One-parameter semigroups for linear evolution equations. Springer-Verlag, Berlin (2000).

11. P. Chernoff. Note on product formulas for operator semigroups // J. Funct. Anal. 2:2, 238-242 (1968).

12. V.I. Bogachev, O.G. Smolyanov. Real and functional analysis: University course. RKhD, Moscow (2009). (in Russian).

13. V.I. Bogachev. Measure theory. V. 1.. RKhD, Moscow (2003). [Springer, Berlin (2007).]

14. M.A. Shubin. Spectral theory of elliptic operators on non-compact manifolds // in "Méthodes semi-classiques Vol. 1. École d'Été". Nantes (1991). Soc. Math. France, Astérisque. 207, 35-108 (1992).

Konstatin Yurievich Zamana,

Moscow Institute of Physics and Technology,

Institutskiy av. 9,

141701, Dolgoprudny, Russia

E-mail: zamana.kyu@phystech.edu 